recognition, and advancement to foster an inclusive, equitable, and diverse research workforce. DRIVEN provides a platform, a community, and a common place where individuals can access resources to more easily identify opportunities aligned with their specific research goals, as well as peer and network support at every step along their professional journey. DRIVEN is uniquely aligned to assist investigators with applying for funding through NIH diversity supplements, foundation opportunities, and other national awards. RESULTS/ANTICIPATED RESULTS: DRIVEN provides networking opportunities, information, and writing support for funding opportunities. Since its inception, less than a year ago, we have seen an increase in writing groups, matched investigators with funding opportunities, and provided networking opportunities for mentors and mentees to meet and for peer mentoring to occur. The interest and momentum surrounding DRIVEN both from internal advisory groups and external advisory groups is significant and will only continue with the endorsement of UAB leadership. DRIVEN is expected to be used as a tool for the recruitment and retention of diverse faculty not only within the UAB community but across the CCTS Partner Network thus changing healthcare in the region. DISCUSSION/SIGNIFICANCE OF IMPACT: According to the NIH, research shows that diverse teams who capitalize on innovative ideas and distinct perspectives outperform less diverse teams. Not only is achieving diversity in the biomedical research workforce critical, but providing diverse researchers with access to support and community is competitive necessity.

4160

\section{Evaluating Student Team Dynamics}

Celia Chao ${ }^{1}$, Emma Tumilty ${ }^{1}$, Celia Chao ${ }^{1}$, Judith Aronson ${ }^{1}$, Jonathan D. Hommel ${ }^{1}$, and Mark R. Hellmich ${ }^{1}$

${ }^{1}$ University of Texas Medical Branch

OBJECTIVES/GOALS: We aimed to explore the students' assessments of workload distribution by comparing personal reflective commentaries and team documents defining division of labor in a team science setting. METHODS/STUDY POPULATION: The Interprofessional Research Design course models the team science experience by bringing together $\mathrm{MD}$ and $\mathrm{PhD}$ students to write a research grant. Four teams of 13 students were tasked with both individual and team-based assignments: 1) Each week, each student reported their perception of their own and their team members' effort over the week (totalling 100\%). 2) Iterative work contracts for each team were submitted at four time-points; assigned work toward project completion totalled 100\%. 3) Lastly, each student submitted a short commentary reflecting on the prior week's team dynamics and teamwork. We retrospectively performed a mixedmethods analysis of the workload data. RESULTS/ANTICIPATED RESULTS: Group-reporting in the team contracts remained static throughout the course, often stating equal distribution of workload, whereas individual reporting was more dynamic. Of 13 students, 8 rated more than $50 \%$ of the weeks as balanced. Among some students, there was a discordance of workload distribution when comparing the group document to the individual perceptions of work performed by their teammates. Reflective writing mapped more closely to individual quantitative reports. The data also revealed within team variations, where one student may report a higher proportion of their contributions, while the rest of the team attributed that student a lower percentage of the total work. DISCUSSION/ SIGNIFICANCE OF IMPACT: An important aspect of team function is workload distribution. Group-based workload discussions may be a useful framework, but does not provide insight into team dynamics, whereas individually reported workload distributions and short reflections seem to more accurately inform us on team function.

4468

\section{Evaluating the Emerging Investigators Website as an} Educational Resource for Early Career Researchers Layla Fattah ${ }^{1}$, Inga Peter, PhD ${ }^{1}$, Jenny Lin ${ }^{1}$, and Janice Lynn Gabrilove, MD, FACP ${ }^{1}$

${ }^{1}$ Mount Sinai School of Medicine

OBJECTIVES/GOALS: The aim of this project is to assess the usability and acceptance of a web-based educational resource for early career researchers. The Emerging Investigators website is designed to bring together resources, provide educational support and foster a community of early career researchers throughout the Mount Sinai Health System (MSHS). Locally designed and built, this web-based platform is developed using the principles of Community of Inquiry (COI), which considers how the design of online learning environments might best create and sustain a sense of community among learners. Developing a resource that meets the needs of this cohort of researchers requires an iterative implementation strategy guided by user feedback. A formal website roll-out strategy and accompanied evaluation aims to determine the design, navigability, content, relevance and educational value of this online resource from a user perspective. METHODS/ STUDY POPULATION: In order to ensure this resource effectively meets the needs of this cohort of researchers, a mixed process of evaluation and design was utilized. An initial phase 1 survey was conducted with TL1 and KL2 scholars. Surveys consisted of standardized questions with answers arranged as Likert-type scales and additional written responses to collect valuable qualitative data. A convenience sample of early career researchers at Mount Sinai were contacted for initial survey participation $(\mathrm{N}=10)$. A total of 3 junior faculty KL2 scholars, 3 TL1 post-doc and 4 TL1 pre-doc scholars responded to the survey. Participants were initially asked to comment on design, functionality and usefulness of content on a Likert scale with qualitative comments to support the given scores. They were subsequently asked to consider what key topics or resources were missing from the website. Based on the initial survey, changes were made to the format and content of the Emerging Investigators website to improve content relevance and usability. For phase 2, an evaluation rubric was developed to assess design, navigability, content, relevance, along with three key COI criteria to determine the educational value of this online resource. The rubric will be utilized to collect feedback in the wider phase 2 roll out of the website. RESULTS/ANTICIPATED RESULTS: The first phase of survey feedback shaped overall design of the resource. The second phase will comprehensively evaluate the value of the website in the context of teaching and learning for emerging investigators. Ten surveys were captured in the first phase. Data collection is ongoing for the second phase. Phase 1 feedback was primarily qualitative, and valuable in informing overall design choices and content. Overall the website was well received, with participants commenting on the value of the resource in terms of content and educational value. Participants particularly appreciated the regularly updated calendar function and the links provided to a wide range of resources. Functionality issues, such as broken links, were reported by participants and repaired for phase 2. Further topics of content were identified, and additional links and multimedia resources were added to address this feedback. The second phase evaluation is ongoing with data collection being conducted 
via an evaluation rubric. DISCUSSION/SIGNIFICANCE OF IMPACT: The Emerging Investigators website, developed using the principles of COI provides key learning, reading and resources for early career investigators in a format that is well received by a sample group of early career researchers at Mount Sinai. The website has aimed to address the reported need for communication, collaboration and social interaction with peers and other researchers across the MSHS through the addition of further web-based resources such as a LinkedIn page, a blog to feature research and provide a sounding board for research efforts, and a calendar of events targeted specifically at early career researchers. These were highlighted as areas of particular value by the participants. We anticipate the results of phase 2 rubric-based evaluations will provide actionable data that will lead to further refinement of the website, an optimized interface, and improved usability.

4015

Evaluation of the Impact of a Clinical and Translational Science Predoctoral Program on Post-Graduate Outcomes

Alexandra Joelle Greenberg-Worisek, $\mathrm{PhD}, \mathrm{MPH}^{1}$, Katherine Cornelius, Mayo Clinic ${ }^{1}$, Becca Gas ${ }^{1}$, Carmen Silvano ${ }^{1}$, Karen Marie Weavers, Asst. Professor of Medical Education ${ }^{1}$, Lewis R Roberts ${ }^{1}$, Stephen C Ekker, $\mathrm{PhD}^{1}$, Felicity Enders, $\mathrm{PhD}, \mathrm{MPH}^{1}$, and Anthony Windebank, MD ${ }^{1}$

${ }^{1}$ Mayo Clinic

OBJECTIVES/GOALS: The Mayo Clinic Clinical and Translational Science (CTS) Predoctoral program aims to develop independent researchers capable of leading multi-disciplinary teams to accelerate the translation of discovery to application. Here, we detail the outcomes of our graduates over the past ten years (2010-2019). METHODS/STUDY POPULATION:): A survey was fielded with all CTS graduates whose degrees were conferred since the program's inception to 2019. Items addressed their current position, whether they were still involved in research, what type of research they were involved in, and whether they stayed involved with education. They also submitted a recent $\mathrm{CV}$, from which data were collected about publications and grants. A subset were then contacted for a semistructured interview. Items included questions addressing motivation for pursuing a $\mathrm{PhD}$ in CTS, whether the program prepared them for their current work, gaps they felt they had in training, and whether they felt they were making a difference in the lives of patients. RESULTS/ANTICIPATED RESULTS: Of the 41 alumni, 34 responded (83\% response rate). Of these, 19 (56\%) are at Mayo Clinic, 9 (26\%) work for other academic institutions, and 6 (21\%) do not work for an academic institution. Most have remained in research $(33 / 34,97 \%)$. The majority $(22 / 33,67 \%)$ are involved in clinical research, $30 \%(10 / 33)$ in basic science, and $24 \%(8 / 33)$ in healthcare delivery research. Most $(23 / 34,68 \%)$ are engaged in educational activities. When asked about changes they have led, $67 \%$ $(18 / 27)$ led quality improvement projects and 44\% (12/27) designed a new research method. Several hold leadership positions either in their organization $(12 / 16,75 \%)$ or in a professional organization (10/16, 63\%). DISCUSSION/SIGNIFICANCE OF IMPACT: The CTS Predoctoral program successfully prepares scholars for careers involving clinical and translational research; furthermore, alumni remain in research-oriented careers after graduation. We will continue to gather longitudinal data alumni move forward in their careers.
From Translational to Transformational: Establishing CLIC's Vision for a Research Education and Training Community

Alfred Vitale ${ }^{1}$, Russell Lackey ${ }^{1}$, Melissa Trayhan ${ }^{2}$, and Robert White ${ }^{2}$ ${ }^{1}$ University of Rochester Medical Center; ${ }^{2}$ Center for Leading Innovation and Collaboration (CLIC)

OBJECTIVES/GOALS: The new CLIC Education \& Career Development Gateway aims to be a translational science workforce ecosystem for CTSAs to share learning and training resources and career opportunities. The Gateway also provides individualized assistance to identify and implement TS learning and training resources. METHODS/STUDY POPULATION: The CLIC Education \& Career Development Gateway, located on the CLIC website, is an entry way to: 1) the Education Clearinghouse, a platform where CTSA Program hubs can find and share educational resources individually or as part of resource kits; 2) the Opportunities Board, which includes jobs and mini-sabbaticals from CTSA Program hubs; and 3) the Education \& Training Navigator, a personalized approach to education and training requests. These approaches help empower and support a cooperative learning and training community that is inclusive and collaborative, facilitating and amplifying opportunities for the sharing of educational resources throughout the translational science workforce. RESULTS/ ANTICIPATED RESULTS: Through a person-centered, direct engagement approach, the anticipated outcomes of these efforts are to promote increased collaboration across CTSA Program Hubs and partners, and the amplification of accessible, relevant existing resources. Another anticipated outcome is increased production of educational materials through the reduction of work duplication and identification of gaps in education and training resources. The Gateway also provides an opportunity to communicate the work and efforts that consortium-level special groups (working groups, special interest groups, etc.) produce. Ongoing evaluations and suggestions will help determine future improvements and functionalities. DISCUSSION/SIGNIFICANCE OF IMPACT: CLIC's education and training ecosystem promotes education as a community space to facilitate opportunities for collaboration and partnerships, amplifying visibility of the work created by members of the CTSA community, and encouraging a transformative career trajectory for trainees and scholars.

4143

\section{HiREC Endowment: Building Models in Research Capacity for Infrastructure Sustainability and Productivity \\ Lourdes E. Soto de Laurido ${ }^{1}$, Walter R. Frontera ${ }^{1}$, and Aracelis Huertas ${ }^{1}$ \\ ${ }^{1}$ University of Puerto Rico, Medical Sciences Campus}

OBJECTIVES/GOALS: Improve infrastructure, resources, partnerships, and metrics to enhance the research environment for Hispanic researchers as a Minority Serving Institution. To support the research infrastructure in our Campus to encourage a research culture of sustainability and productivity. METHODS/STUDY POPULATION: Development of four research capacity-building models to enhance the pathway of junior researchers as independent researchers:1. MSc Phase I-Scholar Award 2 years in a Post Doctoral Master in CTR ; 2 . Advanced CTR Award 1 year to support research 